\title{
A Framework for Constraint Based Local Search using ESSENCE
}

\author{
Özgür Akgün, Saad Attieh, Ian P. Gent, Christopher Jefferson, Ian Miguel, \\ Peter Nightingale, András Z. Salamon, Patrick Spracklen, James Wetter \\ School of Computer Science, University of St Andrews, UK \\ \{ozgur.akgun, sa74, ian.gent, caj21, ijm, pwn1, Andras.Salamon, jlps\}@ st-andrews.ac.uk
}

\begin{abstract}
Structured Neighbourhood Search (SNS) is a framework for constraint-based local search for problems expressed in the ESSENCE abstract constraint specification language. The local search explores a structured neighbourhood, where each state in the neighbourhood preserves a high level structural feature of the problem. SNS derives highly structured problem-specific neighbourhoods automatically and directly from the features of the ESSENCE specification of the problem. Hence, neighbourhoods can represent important structural features of the problem, such as partitions of sets, even if that structure is obscured in the low-level input format required by a constraint solver. SNS expresses each neighbourhood as a constrained optimisation problem, which is solved with a constraint solver. We have implemented SNS, together with automatic generation of neighbourhoods for high level structures, and report high quality results for several optimisation problems.
\end{abstract}

\section{Introduction}

Constraint Programming (CP) offers an efficient means of solving complex constrained optimisation problems. A systematic search through the space of partial assignments may be employed to achieve this. In local search methods [Hoos and Stützle, 2004], a complete assignment is iteratively modified in an effort to reach a satisfying or optimal solution. At each iteration a modification is selected from a neighbourhood of available candidates. Local search sacrifices completeness for the ability to explore neighbourhoods rapidly.

Constraint-based local search (CBLS) [Codognet and Diaz, 2001; Van Hentenryck and Michel, 2005] has applied local search to CP. Neighbourhoods are specified by the user as part of the model, or generated from the constraints and variables in the model [Benoist et al., 2011; Björdal et al., 2015]. A potential weakness of this approach is that a constraint model typically represents the abstract structure of a given problem as a collection of primitive variables and constraints. Focusing on variables and constraints individually may produce weaker neighbourhoods than if the original problem structure were apparent. This observation motivates our work: by allowing neighbourhoods to exploit the original problem's combinatorial structure, we expect local search to attain better performance than when problem structure is not available.

The technique of Large Neighbourhood Search (LNS) originated in the constraint community [Shaw, 1998], where it has been further refined to exploit propagation [Perron et al., 2004] and explanations [Prud'homme et al., 2014]. There are also customisable and hybrid LNS approaches [Rendl et al., 2015; Cipriano et al., 2013]. Pesant and Gendreou extended LNS to support meaningful neighbourhoods (e.g. exchanging two cities in the Travelling Salesperson Problem) [1999]. The advance we present here is that SNS is able to automatically generate neighbourhoods from a high level specification which are semantically meaningful in the original problem.

For high level problem expression we use the ESSENCE constraint specification language [Frisch et al., 2005; 2007; 2008]. Space precludes a detailed presentation, but Fig. 1 shows an illustrative specification of the capacitated vehicle routing problem (CVRP). An ESSENCE specification identifies: the input parameters of the problem class (given), whose values define an instance; the combinatorial objects to be found (find); the constraints the objects must satisfy (such that); identifiers declared (letting); and an (optional) objective function ( $\mathrm{min} / \mathrm{maximising}$ ). Here, the combinatorial object to be found is represented by a single abstract variable whose type is set of sequence of int. This is a considerably higher level of description than previous work on CBLS described as working from high-level models [Van Hentenryck and Michel, 2007]. Ågren et al. extend local search to support sets of integers directly [2009]. We improve over this by supporting arbitrarily nested types. Furthermore, we avoid having to add explicit support for these types to the underlying constraint solver.

ESSENCE is situated at the top of a constraint modelling pipeline, which automatically models and solves an ESSENCE specification. The CONJURE automated modelling system [Akgün et al., 2011; 2013] employs refinement rules to convert an ESSENCE specification into a lowerlevel but generic language ESSENCE PRIME [Rendl, 2010]. This model is automatically prepared for a given constraint solver while performing solver-specific model optimisations by SAVILE Row [Nightingale et al., 2014; 2015; 2017].

By using ESSENCE as the starting point we can exploit the structure in ESSENCE specifications, avoiding the diffi- 


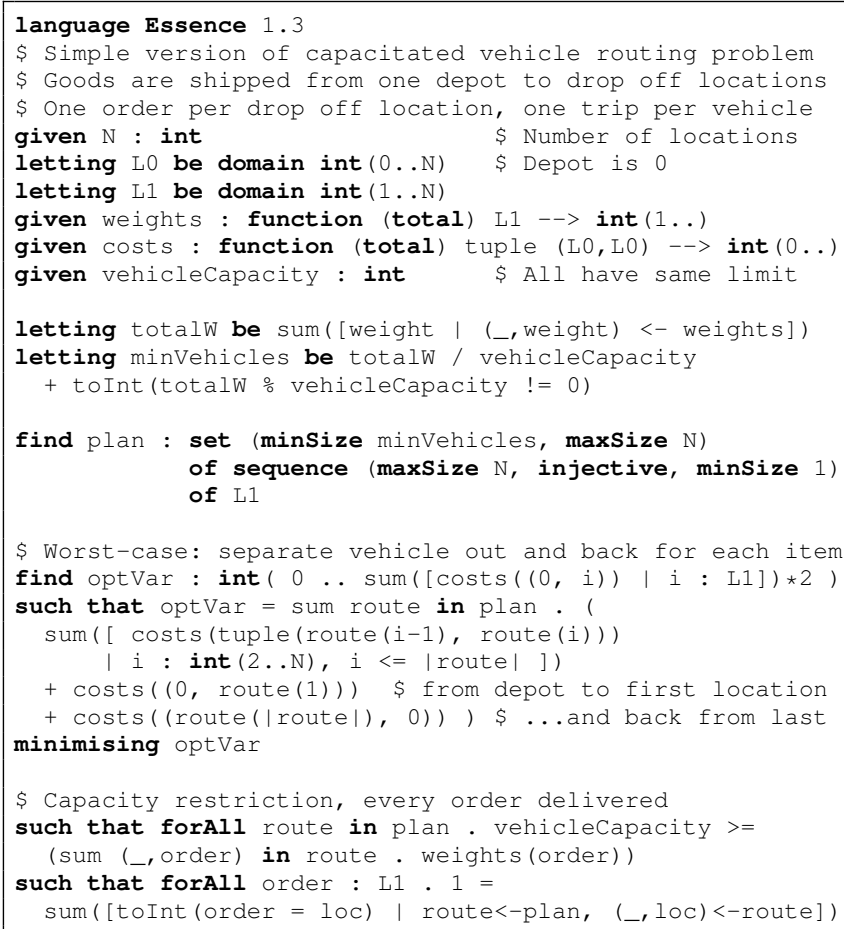

Figure 1: Capacitated vehicle routing in ESSENCE

cult task of identifying that structure in an equivalent constraint model. Neighbourhoods are automatically generated as constraint problems describing transformations of members of ESSENCE domains via a library of domain specific operations that encapsulate expert knowledge. The resulting method, which we call Structured Neighbourhood Search, retains the advantages of specifying problems in ESSENCE concision, abstraction from modelling details and blackbox search - while actually improving the quality of the neighbourhoods we can generate for constraint-based local search.

\section{An Overview of SNS}

To help the reader follow our exposition we start with a highlevel overview of Structured Neighbourhood Search. The key point is that the use of ESSENCE allows neighbourhoods explored by the low-level search to be semantically meaningful in the high-level specification of the problem. For example, in a problem concerning sets, SNS can ensure that consecutive states in the local search represent valid sets, even using a low-level solver which is not aware of the structure of sets. To achieve this, CONJURE analyses a specification and automatically generates semantically meaningful neighbourhoods (Sec. 3). The pipeline then refines these neighbourhoods so that they can be used by the SNS solver. The SNS solver (Sec. 4) then performs a relatively standard local search, selecting appropriate neighbourhoods automatically using a multi-armed bandit algorithm (Sec. 4.4). Within each neighbourhood, a complete constraint solver is used to ensure that the structural constraints defining the neighbourhood are obeyed. As a result, SNS combines the large neighbourhoods of LNS, the high-level semantic structure (without the need for a specially-written search procedure), and the efficiency of modern constraint solvers at the low-level.

\section{Automatic Neighbourhood Generation}

To date, our pipeline has targeted systematic constraint and SAT solvers as output [Nightingale et al., 2017]. In this paper we describe modifications to the pipeline to support SNS. Our main contribution is that for each abstract decision variable in the original specification, a set of neighbourhoods is generated automatically according to a set of neighbourhood generation rules. The strength of these neighbourhoods is that they encode powerful operations derived from the structure of the abstract variable, which provide strong guidance for the SNS search process. These neighbourhoods would be both difficult and costly to generate from the equivalent collection of primitive variables and constraints if a constraint model were the starting point because the abstract structure (e.g. a sequence, set or function) would have to be recognised.

The CVRP specification in Fig. 1 uses a single abstract variable plan, modelled as a set of sequence of int. Neighbourhoods we generate for this variable include: add or remove sequences to/from the set; select a sequence from the set and reverse a contiguous subsequence; select two sequences $s_{1}$ and $s_{2}$ and for a range of indices, swap the values contained by $s_{1}$ at those indices with the values contained by $s_{2}$ at the same indices. This example illustrates the advantage of SNS: the set of sequence of int type is explicit in the ESSENCE specification, and can be exploited to automatically generate high quality neighbourhoods.

A neighbourhood specification extends the original ESSENCE specification of a problem with additional abstract variables and constraints to express each of the neighbourhoods to be explored for this problem. Each such neighbourhood is generated automatically by CONJURE. We illustrate this process by means of a concrete example, focusing on the variable type set of sequence of int and the neighbourhood mentioned above: select a sequence from the set and reverse a contiguous subsequence.

We refer to the abstract variables in the original ESSENCE specification as primary variables. Each neighbourhood is expressed as a set of constraints relating these primary variables to an active solution, which is a feasible solution to the problem. The first step in neighbourhood generation is for each primary variable to introduce a corresponding active variable to store the currently active solution. For the example given in Fig. 1, CONJURE introduces a new active variable active_plan corresponding to the primary variable plan:

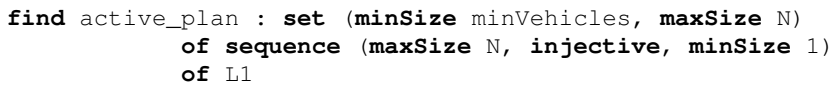

The next step is to add variables local to each neighbourhood. All neighbourhoods have an associated size variable, which control the size of the neighbourhood explored. Further variables may be introduced to control other such parameters. In our example, an additional variable is introduced to indicate the starting index of the subsequence to be reversed:

find revSubSize : int $(1 \ldots N)$

find substart : int $(1 \ldots N)$ 
The final step is to express each neighbourhood by adding constraints between primary and active variables. In our example below, CONJURE adds constraints to the specification constraining the value of the primary variable plan to be the same as that held by active_plan except that one of the sequences in plan must have a contiguous portion reversed.

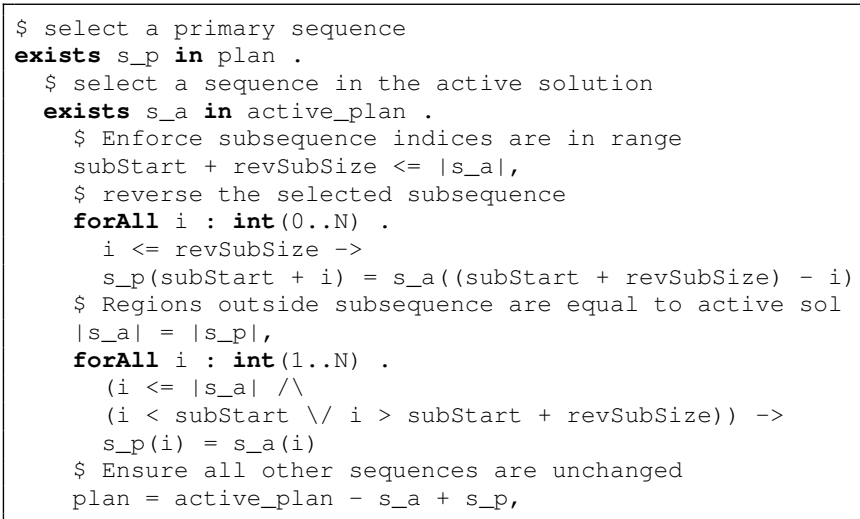

There are typically a variety of neighbourhoods in each neighbourhood specification. In order that the SNS search process can choose which to employ at any particular time, each is enabled with a Boolean activator variable.

\subsection{Neighbourhood Generation Rules}

Our neighbourhood generation rules are driven by the highlevel nested structure of ESSENCE types. Direct rules (Table 1) are concerned solely with the outer structure of an ESSENCE decision variable. For instance, we might wish to reverse a contiguous subsequence of a sequence of $\tau$, where $\tau$ is any ESSENCE type. Lifted rules are parameterised by other neighbourhood generation rules. They allow us to reach inside a containing abstract structure and apply other rules to manipulate individual parts of that structure. This was illustrated in our example, where we selected and reversed a subsequence of a single sequence from a set of sequences.

Lifted rules can themselves be lifted, allowing us to generate a large variety of neighbourhoods by composing a small number of neighbourhood generation rules in multiple ways.

Powerful neighbourhoods can be constructed to make multiple simultaneous changes, which must be coordinated so that the modifications made are valid. For example, given a set of sequences we may wish to exchange elements between sequences, hence modifying the value of two sequences within the set simultaneously. These Synchronised rules (Table 2) can both operate directly or be lifted.

\section{Structured Neighbourhood Search}

The process described in the previous section generates a neighbourhood specification, which is automatically refined by CONJURE into a constraint model in ESSENCE PRIME. ESSENCE PRIME is a solver-independent constraint modelling language offering features similar to those of MiniZinc [Nethercote et al., 2007] or OPL [Van Hentenryck et al., 1999], with support for Boolean and integer decision variables together with arithmetic, logical and global constraints. This model is annotated by CONJURE to indicate the set (and multiset)

Remove $s$ elements from set.

Add $s$ elements to set.

Exchange $s$ elements for values not currently in set.

ExchangeOrRemove a total of $s$ elements from set.

ExchangeOrAdd a total of $s$ elements to set.

sequence

Reverse contiguous subsequence of length $s$.

Exchange the positions of $s$ elements with another $s$.

Relax domains of contiguous subsequence, length $s$.

$\mathbf{C y c l e L / R}$ elements of a sequence $s$ positions left/right.

RemoveE/S $s$ elements from the end/start of a sequence.

AddE/S $s$ elements to the end/start of a sequence.

function

Define values for $s$ more elements of the function domain.

UnDefine values for $s$ elements of the function domain.

Injective- Select $2 s$ elements from the image of a function: reduce to $s$ elements by constraining pairs of elements in the preimage to be equal.

Injective+ Select $s$ elements from the image of a function: increase to $2 s$ elements by constraining pairs of elements in the preimage to be distinct.

Permute the images of $s$ inputs to the function.

Table 1: Direct neighbourhood generations rules for size $s$. Each of these can be Lifted to operate on nested types.

set (and multiset)

Move $s$ elements from one set to another.

Exchange $s$ elements between two sets.

Merge a set with another set of size $s$.

Split $s$ elements from a set to create a new set.

sequence

Concatenate one sequence with another.

Exchange $s$ elements between two sequences.

Split $s$ elements from a sequence to create a new sequence.

CrossOver Exchange $s$ contiguous elements between two sequences at the same indices.

Move Remove $s$ elements from the end of a sequence; add those elements to the start or end of another sequence.

function

CrossOver Exchange $s$ elements from the image of one function with $s$ elements from the image of another.

Table 2: Synchronised neighbourhood generation rules for size $s$. These rules can be used directly on a single variable or can be lifted to operate on parts of an abstract structure.

parts corresponding to each neighbourhood then processed by SAVILE Row into input suitable for the SNS solver. 


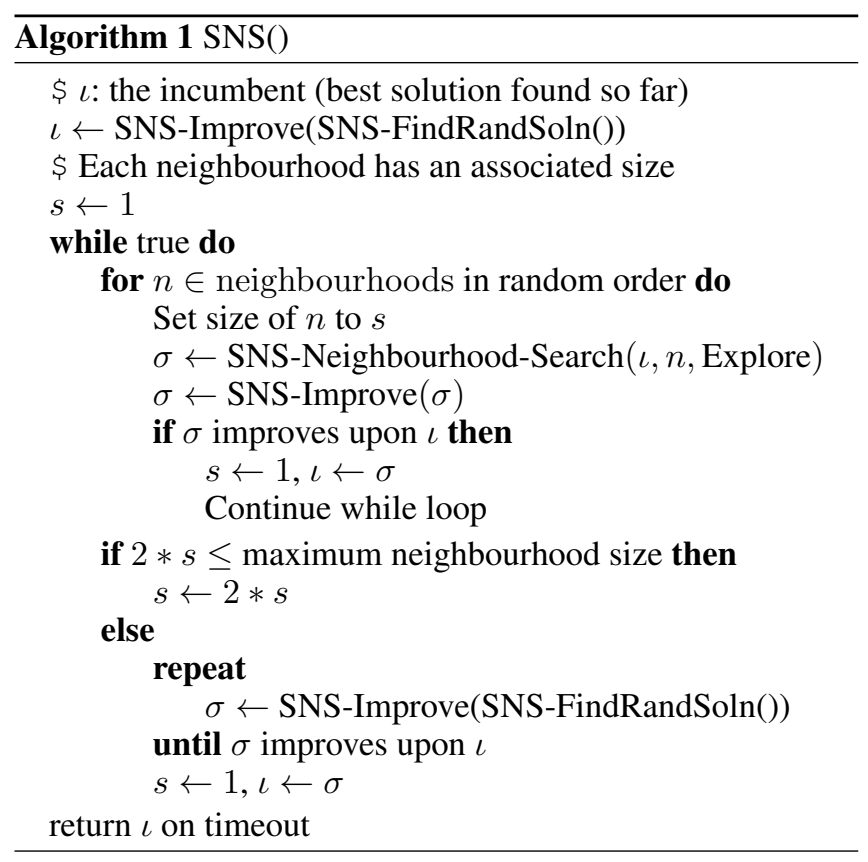

\subsection{The SNS Search Procedure}

Algorithm 1 summarises the SNS search procedure. The first step is to find a random feasible solution ( $\$ 4.2)$. The random feasible solution is improved by applying SNS-Improve() and it becomes the first incumbent solution $\iota$. The variable $s$ represents the neighbourhood size used for diversification, and $s=1$ initially. The first part of the main loop picks a neighbourhood and applies it to $\iota$ (with size $s$ ) to move away from $\iota$, creating a new active solution $\sigma$ that may be worse than $\iota$. SNS-Improve() is applied to improve $\sigma$. If the result is better than $\iota$ it is accepted as the new incumbent, and $s$ is reset to 1 .

In the case where the set of neighbourhoods is exhausted, $s$ is increased and each neighbourhood is applied again. The diversification process is repeated until $s$ cannot be increased further. Finally, the algorithm will generate new random feasible solutions (unrelated to $\iota$ ), apply SNS-Improve() to each one, and accept the first that improves on $\iota$. In the following subsections we describe the constituent parts of the algorithm.

\subsection{Finding Random Feasible Solutions}

At two points $S N S($ ) requires the production of random feasible solutions (function SNS-FindRandSoln()): to find the initial incumbent and to escape local optima when the maximum neighbourhood size has been reached. For this purpose, we employ the MINION constraint solver [Gent et al., 2006] with the $\mathrm{dom} / w d e g$ variable ordering [Boussemart et al., 2004], a random value ordering and random restarts, beginning at $100 \mathrm{~ms}$ and increasing by a multiple of 1.5 each iteration.

\subsection{Finding Improved Solutions}

Algorithm 2 SNS-Improve() is called with a feasible solution $\sigma$, the active solution. We select a neighbourhood (§4.4) and run the neighbourhood search procedure $(\S 4.5)$ in optimisation mode. If a solution $\sigma^{\prime}$ that is at least as good as $\sigma$ is found, $\sigma^{\prime}$ is made the new active solution and the process is repeated. If no $\sigma^{\prime}$ of sufficient quality is found within a given

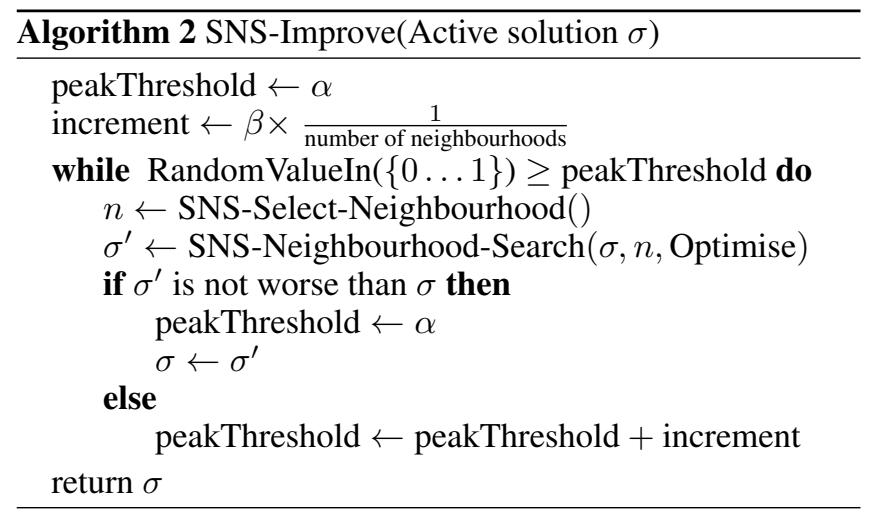

timeout, we add an increment to the peakThreshold, which is used to decide probabilistically if we have reached a local optimum. In this paper we set $\alpha$ to 0.001 and $\beta$ to $\frac{1}{16}$. Future work will explore tuning these parameters.

\subsection{Neighbourhood Selection}

The SNS-Select-Neighbourhood() procedure (called from Algorithm 2) is used to find the most promising neighbourhood at any point during search. For a given problem there may be many generated neighbourhoods for which we have no prior knowledge of their reward distributions, determined by how much the application of each neighbourhood improves the objective. As we have no prior knowledge of the suitabilities of particular neighbourhoods to a particular problem class, during search we have to carefully balance the time taken exploring and identifying a neighbourhood's performance while exploiting those that allow us to rapidly improve. Representing this as a multi-armed bandit problem allows us to employ well known regret minimising algorithms to deal with the exploration/exploitation dilemma. This can be seen as a form of 'Adaptive LNS' [Ropke and Pisinger, 2006].

The multi-armed bandit can be seen as a set of real distributions, each distribution being associated with the rewards delivered by one of the $K$ levers. In our case this is the $K$ generated neighbourhoods. On each iteration of SNS-Improve() one neighbourhood is selected to locally search the problem space and a reward is observed based upon the improvement to the active solution. Our aim is at each iteration to apply the optimal neighbourhood, where optimality is defined as producing the largest increase in the value of the objective. The regret $\rho$ after $T$ rounds is defined as the expected difference between the reward sum associated with an optimal strategy and the sum of the collected rewards observed. The UCB1 [Auer et al., 2002] algorithm was chosen to solve the multi-armed bandit problem as first and foremost its regret grows logarithmically in line with the number of actions taken. For each neighbourhood $k$ we record the average reward $x^{k}$ and the number of times $k$ has been tried in the search procedure $n_{j}$ out of a total of $n$ iterations. On each iteration a neighbourhood is chosen that maximises $x_{j}+\sqrt{2 \log (n) / n_{j}}$.

In our system the reward distributions for a neighbourhood are not fixed, so this is not a Stationary Multi-Armed Bandit problem. However, if a neighbourhood performs well, we expect it will continue performing well during search even if there is a slight variation in the mean reward. We have 
found that using UCB1 gives good results. Future work could investigate the use of Upper Confidence Bound policies for non-stationary bandit problems, such as the family of Exp3 algorithms [Kocsis and Szepesvári, 2006; Munos, 2014].

\subsection{Neighbourhood Search}

SNS-Neighbourhood-Search $(\sigma, n$, Mode) is responsible for searching the neighbourhood $n$ of the active solution $\sigma$. It can be run in two Modes. The first (Explore) accepts the first solution of any objective value. This is used by $S N S($ ) in Algorithm 1. The second mode (Optimise, employed by SNSImprove()) treats the neighbourhood as a constrained optimisation problem, accepting only those solutions with an objective value at least as good as that of the active solution $\sigma$. Once Optimise finds a solution, it will continue to search for a solution with better objective value, until the time limit of $500 \mathrm{~ms}$ is reached. In preliminary experiments we found a time limit more effective than a search node limit as the latter penalises simple neighbourhoods that have high node rates.

Neighbourhood search is performed using the systematic MINION solver in a carefully controlled manner. The annotations supplied by CONJURE are used to identify the primary and active variables involved in neighbourhood $n$. The primary variables are unassigned preparatory to search, and the activator variable for neighbourhood $n$ is set to true, removing the guard on the neighbourhood constraints.

We construct a variable ordering as follows. First is the neighbourhood size variable for $n$, which is given an ascending value ordering. This forces the exploration to begin close to the current active solution $\sigma$ before systematically expanding out. Next are placed the variables that control the neighbourhood parameters, e.g. subStart in our example. A random value ordering is used so that different portions of the neighbourhood are searched on each activation. Finally come the primary variables upon which the neighbourhood is operating, that is the representation of a partition, set, sequence, etc. These variables are searched using a dom/wdeg ordering with the aim of finding solutions as quickly as possible once the neighbourhood parameters have been chosen.

\section{Experimental Evaluation}

Our hypothesis is that the structure in abstract ESSENCE specifications can be exploited to automatically generate effective neighbourhoods for constraint-based local search. The most natural comparison is with methods that generate neighbourhoods from the constraint model. Therefore we compare SNS with OscaR/CBLS [Björdal et al., 2015], a modern implementation of CBLS, propagation-guided LNS (LNS PG) [Perron et al., 2004], and explanation-based LNS (LNS EB) [Prud'homme et al., 2014] (which are automatic large neighbourhood search algorithms). LocalSolver [Benoist et al., 2011] is another possible point of comparison, but this commercial solver incorporates a complex mix of technologies that make it unsuitable to test our hypothesis. We use Chuffed (via its free search option), a systematic lazy clause generation solver [Chu, 2016], as a performance baseline.

We compare SNS to the other solvers on 5 problem classes: Capacitated Vehicle-Routing Problem (CVRP); Progressive
Party Problem (PPP, CSPLib 13); Minimum Energy Broadcast (MEB, CSPLib 48); SONET (CSPLib 56); Rack Configuration Problem (RackConf, CSPLib 31).

For SNS, each problem class and a set of instances is specified in ESSENCE. An extended version of CONJURE is applied once per problem class to generate a model in ESSENCE PRIME with SNS neighbourhoods. An extended version of S AVILE Row [Nightingale et al., 2017] is applied for each instance to instantiate the model and specialise it in two ways: SNS (including neighbourhoods) and MiniZinc (with neighbourhoods removed). The MiniZinc instance is then specialised via MiniZinc 2.1.7 for OscaR/CBLS and Chuffed.

For the LNS methods, each problem class was implemented in Choco 4.0.6, with great care taken to match the ESSENCE PRIME model as closely as possible. For the search within each LNS neighbourhood we use dom/wdeg variable ordering [Boussemart et al., 2004], ascending value ordering and a limit of 50 backtracks (a value found to be good in preliminary experiments). To find the first solution we replicate the method in $\S 4.2$ in Choco, except that the time limit is initially 1 second rather than $100 \mathrm{~ms}$.

SNS, OscaR/CBLS and the LNS methods are randomised while Chuffed is deterministic. For each solver and instance we perform 10 runs each with a 10 minute time limit. Experiments were run on an Intel Xeon E5-2640 v4 at 2.40GHz with 20 cores (40 hyper-threads) with 20 processes run in parallel.

Table 3 summarises our results. SNS is extremely effective: this is indicated by the proliferation of ' 1 ' entries in the first column of SNS results, indicating that SNS found the best results (or tied) of any competing solver on those instances. Also, SNS was the only solver to successfully find a solution in every run (indicated by the absence of $\infty$ 's in the second column). The two LNS variants are also effective, but SNS is more effective overall. SNS outperforms the LNS variants on all CVRP and SONET instances. On mean best score found, for CVRP the wins are by $6.7 \%$ (EB) and 19\% (PG), while for SONET the wins are by $10 \%$ (EB) and $0.2 \%$ (PG). On PPP, SNS is $0.4 \%$ better than LNS EB, and $3.4 \%$ worse than LNS PG. On the MEB instances, SNS is $2.7 \%$ worse than LNS EB, and $4.5 \%$ better than LNS PG. Both LNS variants outperform SNS on the RackConf instances, although by only $0.2 \%$.

The problem with the most structure in the variables, CVRP, is one where SNS performs particularly well. CVRP is specified with a single decision variable, but it has a nested type: a set of sequences of integers. SNS generates both lifted and direct neighbourhoods of sequences and it substantially outperforms all other solvers. Our results bear out our hypothesis: specifications can be exploited to automatically generate effective neighbourhoods for constraint-based local search.

\section{Conclusion and Further Work}

Structured Neighbourhood Search (SNS) exploits abstract structure in constraint specifications automatically to construct neighbourhoods that reflect that structure, and then performs local search. Our implementation outperforms variants of Large Neighbourhood Search (LNS) and Constraintbased Local Search (CBLS), especially on instances with complex abstract structure. This is particularly notable given 
Proceedings of the Twenty-Seventh International Joint Conference on Artificial Intelligence (IJCAI-18)

\begin{tabular}{|c|c|c|c|c|c|c|c|c|c|c|c|}
\hline Instance & opt & & & OscaR & CBLS & Chu & fed & $\mathrm{LN}$ & EB & $\mathrm{LN}$ & PG \\
\hline CVRP-1 & 290 & 1 & 1.002 & 1.048 & 1.166 & 1.021 & 1.021 & 1 & 1.001 & 1 & 1 \\
\hline CVRP-2 & 375 & 1 & 1.025 & 1.808 & $\infty$ & 2.216 & 2.216 & 1.101 & 1.147 & 1.104 & 1.255 \\
\hline CVRP-3 & 569 & 1 & 1.077 & 2.058 & $\infty$ & 2.162 & 2.162 & 1.104 & 1.181 & 1.336 & 1.434 \\
\hline CVRP-4 & 529 & 1 & 1.179 & $\infty$ & $\infty$ & 3.837 & $\infty$ & 1.129 & 1.34 & 1.499 & 1.683 \\
\hline CVRP-5 & 114 & 1 & 1 & 1 & 1 & 1 & 1 & 1 & 1 & 1 & 1 \\
\hline PPP-1 & 13 & 1 & 1.054 & $\infty$ & $\infty$ & 1 & 1.008 & 1 & 1.054 & 1 & 1.038 \\
\hline PPP-2 & 12 & 1.167 & 1.258 & $\infty$ & $\infty$ & 1 & 1 & 1.167 & 1.25 & 1.083 & 1.225 \\
\hline PPP-3 & 12 & 1.167 & 1.225 & $\infty$ & $\infty$ & 1.25 & 1.25 & 1.167 & 1.233 & 1 & 1.183 \\
\hline PPP-4 & 13 & 1 & 1.1 & $\infty$ & $\infty$ & 1.077 & 1.077 & 1.077 & 1.146 & 1.077 & 1.177 \\
\hline PPP-5 & 14 & 1 & 1.064 & $\infty$ & $\infty$ & 1 & 1 & 1 & 1.029 & 1 & 1.036 \\
\hline PPP-6 & 13 & 1.077 & 1.123 & $\infty$ & $\infty$ & 1.077 & 1.077 & 1.077 & 1.108 & 1 & 1.131 \\
\hline PPP-7 & 13 & 1 & 1.115 & $\infty$ & $\infty$ & 1 & 1 & 1.077 & 1.162 & 1.077 & 1.085 \\
\hline PPP-8 & 13 & 1 & 1.092 & $\infty$ & $\infty$ & 1.077 & 1.077 & 1 & 1.108 & 1 & 1.092 \\
\hline PPP-9 & 13 & 1.077 & 1.131 & $\infty$ & $\infty$ & 1 & 1 & 1.077 & 1.162 & 1 & 1.092 \\
\hline MEB-01 & 202 & 1 & 1 & 1 & 1.121 & 2.98 & 3.05 & 1 & 1 & 1 & 1 \\
\hline MEI & 205 & 1 & 1.16 & $1.6 ?$ & 2.391 & 5.868 & 5.868 & 1 & 1.001 & 1 & 1.032 \\
\hline MEB-03 & 328 & 1 & 1 & 1 & 1.005 & 1 & 1 & 1 & 1 & 1 & 1 \\
\hline MEB-04 & 160 & 1 & 1 & 1.456 & 1.501 & 1 & 1 & 1 & 1 & 1 & 1 \\
\hline MEB-05 & 52 & 1.423 & 1.869 & 2.038 & $\infty$ & 6.808 & 6.808 & 1 & 1.396 & 1.538 & 1.998 \\
\hline MEB-06 & 206 & 1 & 1 & 1.005 & 1.013 & 2.51 & 2.552 & 1 & 1 & 1 & 1.001 \\
\hline MEB-07 & 542 & 1 & 1.076 & 1.083 & 1.607 & 2.616 & 2.616 & 1.017 & 1.05 & 1.015 & 1.06 \\
\hline MEB-08 & 581 & 1 & 1.039 & 1.093 & 1.308 & 3.036 & 3.146 & 1 & 1.019 & 1.048 & 1.102 \\
\hline MEB-09 & 344 & 1 & 1.251 & 1.23 & 1.368 & 2.712 & 2.712 & 1.067 & 1.151 & 1.067 & 1.414 \\
\hline MEB-10 & 397 & 1 & 1.021 & 1.06 & 1.597 & 2.499 & 2.499 & 1 & 1.008 & 1 & 1.012 \\
\hline MEB-11 & 466 & 1 & 1.088 & 1.182 & 1.223 & 2.622 & 2.681 & 1.036 & 1.134 & 1.137 & 1.182 \\
\hline MEB-12 & 264 & 1.0 & 1.203 & 1.455 & 1.659 & 6.242 & 6.245 & 1 & 1.297 & 1.277 & 1.596 \\
\hline MEB-13 & 435 & 1 & 1.087 & 1.039 & 1.147 & 1.766 & 1.766 & 1 & 1 & 1 & 1.054 \\
\hline SONET-1 & 59 & 1 & 1.027 & 1.068 & 1.224 & 3.407 & 3.414 & 1.051 & 1.093 & 1.136 & 1.269 \\
\hline SONET-2 & 115 & 1 & 1.058 & 1.13 & 1.421 & 6.417 & 6.417 & 1.07 & 1.233 & 1.009 & 1.067 \\
\hline SONET-3 & 89 & 1 & 1.035 & 1.112 & 1.307 & 4.551 & 4.551 & 1.112 & 1.175 & 1.022 & 1.093 \\
\hline SONET-4 & 122 & 1 & 1.089 & 1.082 & 1.234 & 5.549 & 5.557 & 1.213 & 1.287 & 1.074 & 1.136 \\
\hline SONET-5 & 167 & 1 & 1.169 & 1.054 & 1.314 & 5.491 & 5.491 & 1.18 & 1.283 & 1.024 & 1.066 \\
\hline SONET-6 & 172 & 1 & 1.1 & 1.0 & 1.324 & 5.1 & 5.1 & 1.215 & 1.392 & 1.029 & 1.117 \\
\hline TT-7 & 211 & 1.1 & & 1 & 1.305 & 5.744 & 5.744 & 1.341 & $\infty$ & 1.142 & $\infty$ \\
\hline SONET-8 & 195 & 1.292 & 1.525 & 1 & 1.276 & 7.959 & 7.959 & 1.19 & $\infty$ & 1.056 & $\infty$ \\
\hline RackConf-C & 550 & 1 & 1 & 1 & 1 & 1 & 1 & 1 & 1 & 1 & 1 \\
\hline RackConf-02 & 1100 & 1 & 1 & 1.091 & 1.259 & 1 & 1 & 1 & 1 & 1 & 1 \\
\hline RackConf-03 & 1200 & 1 & 1 & 1.583 & 1.725 & 1 & 1 & 1 & 1 & 1 & 1 \\
\hline RackConf-04 & 1150 & 1 & 1 & 1.0 & 1.061 & 1 & 1 & 1 & 1 & 1 & 1 \\
\hline RackConf-05 & 987 & 1.0 & 1. & 1.0 & 1.074 & 1. & 1.0 & 1 & & 1.001 & 1.001 \\
\hline RackConf-06 & 624 & 1 & 1.017 & $\infty$ & $\infty$ & 1 & 1 & 1 & 1.004 & 1 & 1 \\
\hline RackConf-07 & 664 & 1.011 & 1.016 & $\infty$ & $\infty$ & 1.018 & 1.018 & 1 & 1.006 & 1 & 1 \\
\hline RackConf-08 & 711 & 1.003 & 1.015 & $\infty$ & $\infty$ & 1.025 & 1.025 & 1 & 1.005 & 1 & 1 \\
\hline RackConf-09 & 753 & 1.005 & 1.009 & $\infty$ & $\infty$ & 1.04 & 1.041 & 1 & 1.005 & 1 & 1 \\
\hline RackConf-10 & 783 & 1.005 & 1.018 & $\infty$ & $\infty$ & 1.033 & 1.033 & 1 & 1.019 & 1 & 1.003 \\
\hline RackConf-11 & 584 & 1 & 1.003 & 1.082 & 1.12 & 1.051 & 1.052 & 1 & 1.014 & 1 & 1.002 \\
\hline
\end{tabular}

Table 3: Column opt lists the best value found by any solver. For each algorithm, the first column gives the ratio of the best value found to opt (lower is better with 1 best possible); $\infty$ indicates that no run yielded a solution. The second column gives the ratio of the mean of the best values found at the end of each run to opt (lower is better); $\infty$ indicates at least one run during which no solution was found.

the automatic generation of neighbourhoods from a very high level specification of a constraint problem. Future research includes optimising SNS parameters, or investigating more efficient exploration of the automatically constructed neighbourhoods.

\section{Acknowledgements}

We thank Nguyen Dang for her assistance with the experiments, and EPSRC grants EP/P015638/1 and EP/P026842/1 for funding. Christopher Jefferson is supported by a Royal Society University Research Fellowship.

\section{References}

[Akgün et al., 2011] Özgür Akgün, Ian Miguel, Chris Jefferson, Alan M. Frisch, and Brahim Hnich. Extensible automated constraint modelling. In AAAI, pages 4-11, 2011.

[Akgün et al., 2013] Özgür Akgün, Alan M. Frisch, Ian P. Gent, Bilal S. Hussain, Chris Jefferson, Lars Kotthoff, 
Ian Miguel, and Peter Nightingale. Automated symmetry breaking and model selection in Conjure. In $C P$, pages 107-116, 2013.

[Auer et al., 2002] Peter Auer, Nicolò Cesa-Bianchi, and Paul Fischer. Finite-time analysis of the multiarmed bandit problem. Machine Learning, 47(2):235-256, May 2002.

[Benoist et al., 2011] Thierry Benoist, Bertrand Estellon, Frédéric Gardi, Romain Megel, and Karim Nouioua. LocalSolver 1.x: a black-box local-search solver for 0-1 programming. 4OR, 9(3):299-316, 2011.

[Björdal et al., 2015] Gustav Björdal, Jean-Noël Monette, Pierre Flener, and Justin Pearson. A constraint-based local search backend for MiniZinc. Constraints, 20(3):325-345, 2015.

[Boussemart et al., 2004] Frédéric Boussemart, Fred Hemery, Christophe Lecoutre, and Lakhdar Sais. Boosting systematic search by weighting constraints. In ECAI, pages 146-150, 2004.

[Chu, 2016] Geoffrey Chu. Chuffed, a lazy clause generation solver, 2016. Available from https://github. com/chuffed/chuffed.

[Cipriano et al., 2013] Raffaele Cipriano, Luca Di Gaspero, and Agostino Dovier. A multi-paradigm tool for large neighborhood search. In Hybrid Metaheuristics, pages 389-414, 2013.

[Codognet and Diaz, 2001] Philippe Codognet and Daniel Diaz. Yet another local search method for constraint solving. In SAGA, LNCS 2264, pages 73-90. Springer, 2001.

[Frisch et al., 2005] Alan M. Frisch, Matthew Grum, Chris Jefferson, Bernadette M. Hernández, and Ian Miguel. The Essence of Essence. Modelling and Reformulating Constraint Satisfaction Problems, pages 73-88, 2005.

[Frisch et al., 2007] Alan M. Frisch, Matthew Grum, Chris Jefferson, Bernadette M. Hernández, and Ian Miguel. The design of Essence: A constraint language for specifying combinatorial problems. In IJCAI, pages 80-87, 2007.

[Frisch et al., 2008] A. M. Frisch, W. Harvey, C. Jefferson, B. Martínez-Hernández, and I. Miguel. Essence: A constraint language for specifying combinatorial problems. Constraints 13(3), pages 268-306, 2008.

[Gent et al., 2006] Ian P. Gent, Chris Jefferson, and Ian Miguel. Minion: A fast, scalable, constraint solver. In ECAI, pages 98-102, 2006.

[Hoos and Stützle, 2004] Holger H. Hoos and Thomas Stützle. Stochastic local search: Foundations \& applications. Elsevier, 2004.

[Kocsis and Szepesvári, 2006] Levente Kocsis and Csaba Szepesvári. Bandit based Monte-Carlo planning. In ECML, LNCS 4212, pages 282-293. Springer, 2006.

[Munos, 2014] Rémi Munos. From bandits to Monte-Carlo tree search: The optimistic principle applied to optimization and planning. FTML, 7(1):1-129, 2014.

[Nethercote et al., 2007] Nicholas Nethercote, Peter J. Stuckey, Ralph Becket, Sebastian Brand, Gregory J.
Duck, and Guido Tack. MiniZinc: Towards a standard CP modelling language. In $C P$, LNCS 4741, pages 529-543. Springer, 2007.

[Nightingale et al., 2014] Peter Nightingale, Özgür Akgün, Ian P. Gent, Chris Jefferson, and Ian Miguel. Automatically improving constraint models in Savile Row through associative-commutative common subexpression elimination. In CP, LNCS 8656, pages 590-605. Springer, 2014.

[Nightingale et al., 2015] Peter Nightingale, Patrick Spracklen, and Ian Miguel. Automatically improving SAT encoding of constraint problems through common subexpression elimination in Savile Row. In $C P$, LNCS 9255, pages 330-340. Springer, 2015.

[Nightingale et al., 2017] Peter Nightingale, Özgür Akgün, Ian P. Gent, Christopher Jefferson, Ian Miguel, and Patrick Spracklen. Automatically improving constraint models in Savile Row. Artificial Intelligence, 251:35-61, 2017.

[Perron et al., 2004] Laurent Perron, Paul Shaw, and Vincent Furnon. Propagation guided large neighborhood search. In $C P$, LNCS 3258, pages 468-481. Springer, 2004.

[Pesant and Gendreau, 1999] Gilles Pesant and Michel Gendreau. A constraint programming framework for local search methods. Journal of Heuristics, 5(3):255-279, 1999.

[Prud'homme et al., 2014] Charles Prud'homme, Xavier Lorca, and Narendra Jussien. Explanation-based large neighborhood search. Constraints, 19(4):339-379, 2014.

[Rendl et al., 2015] Andrea Rendl, Tias Guns, Peter J. Stuckey, and Guido Tack. MiniSearch: A solverindependent meta-search language for MiniZinc. In $C P$, LNCS 9255, pages 376-392. Springer, 2015.

[Rendl, 2010] Andrea Rendl. Effective Compilation of Constraint Models. Ph.D. Thesis, Univ. of St Andrews, 2010.

[Ropke and Pisinger, 2006] Stefan Ropke and David Pisinger. An adaptive large neighborhood search heuristic for the pickup and delivery problem with time windows. Transportation Science, 40(4):455-472, 2006.

[Shaw, 1998] Paul Shaw. Using constraint programming and local search methods to solve vehicle routing problems. In CP, LNCS 1520, pages 417-431. Springer, 1998.

[Van Hentenryck and Michel, 2005] Pascal Van Hentenryck and Laurent Michel. Constraint-based local search. MIT Press, 2005.

[Van Hentenryck and Michel, 2007] Pascal Van Hentenryck and Laurent Michel. Synthesis of constraint-based local search algorithms from high-level models. In $A A A I$, pages 273-278. AAAI Press, 2007.

[Van Hentenryck et al., 1999] Pascal Van Hentenryck, Laurent Michel, Laurent Perron, and Jean-Charles Régin. Constraint programming in OPL. In PPDP, LNCS 1702, pages 98-116, 1999.

[Ågren et al., 2009] Magnus Ågren, Pierre Flener, and Justin Pearson. Revisiting constraint-directed search. Information and Computation, 207(3):438 - 457, 2009. 